\title{
Unsual Tubal Twin Ectopic Gestation Report of Three Different Cases
}

\author{
Maheshgir S Gosavi*
}

Siraj Hospital, Thane, India

*Corresponding author: Maheshgir S Gosavi, Siraj Hospital, 4 ${ }^{\text {th }}$ Nizampura, Vanjarpatti Naka, Bhiwandi, Thane, Maharashtra, India, Tel: +91-02522-253200; +91-8693027439; 860587084; Email: raddon84@ rediffmail.com

\section{Abstract}

Gestation outside the uterine cavity in which the implantation occurs in any tissue other than the endometrium is referred as ectopic pregnancy. The most places for occurring ectopic pregnancy ( $97 \%$ of cases) are the fallopian tubes including ampulla $(55 \%)$, isthmus $(25 \%)$, and fimbria (17\%), and in $3 \%$ of patient's ectopic pregnancy occurs in the abdominal cavity, ovary, or cervix. The tubal twin ectopic pregnancy is a rare condition, and the first unilateral tubal twin was reported by De Ott in 1891 , and the first live twin tubal ectopic pregnancy was reported in 1944. A live tubal twin ectopic pregnancy is a very rare condition and among >100 reports of tubal twin pregnancies, till now, only 8 cases were live. Early diagnosis and treatment of women with tubal twin ectopic pregnancy is very important and may decrease the risk of tubal rupture. I present three cases of tubal twin ectopic gestation. In the first case, spontaneous unilateral live tubal twin ectopic gestation. The second and third cases spontaneous ruptured twin ectopic gestation. All three cases were successfully managed and there was no history of assisted reproductive technique fertilization or pelvic inflammatory disease.
\end{abstract}

Keywords: Spontaneous; T tubal twin ectopic; Transvaginal usg

Received Date: June 06, 2018; Accepted Date: June 29, 2018; Published Date: August 7, 2018

\section{Introduction}

Gestation outside the uterine cavity in which the implantation occurs in any tissue other than the endometrium is referred as ectopic pregnancy. The most place for occurring ectopic pregnancy (97\% of cases) is the fallopian tubes including ampulla $(55 \%)$, isthmus $(25 \%)$, and fimbria (17\%), and in 3\% of patient's ectopic pregnancy occurs in the abdominal cavity, ovary, or cervix [1]. The tubal twin ectopic pregnancy is a rare condition, and the first unilateral tubal twin was reported by De Ott [2] in 1891, and the first live twin tubal ectopic pregnancy was reported in 1944. A live tubal twin ectopic pregnancy is a very rare condition and among >100 reports of tubal twin pregnancies, till now, only 8 cases were live [3]. Early diagnosis and treatment of women with tubal twin ectopic pregnancy is very important and may decrease the risk of tubal rupture. The present report describes a successful management of spontaneous twin tubal ectopic pregnancies with no history of assisted reproductive technique fertilization or pelvic inflammatory disease.

\section{Case 1}

Citation: Maheshgir S Gosavi, Unsual Tubal Twin Ectopic Gestation Report of Three Different Cases. J Clin Cases Rep 1(3): 96-103. DOI: https://doi.org/10.46619/joccr.2018.1-1019 
A 35-year-old female, came to casualty at midnight with pain in abdomen for three days with 8 weeks of amenorrhea and

bleeding per vaginum since morning. She had one living issues with full term normal deliveries. She had history of two spontaneous abortions. Upon examination, her vitals were stable. Abdomen examination was unremarkable. Upon per vaginum examination the cervical movement was tender, the uterus was bulky and soft, and bogginess and tenderness were felt in the right adnexa. However, her urine pregnancy test was positive.

Ultrasonography with doppler study showed a uterus of $8.3 \mathrm{~cm} \times 5.4 \mathrm{~cm} \times 4.2 \mathrm{~cm}$ with endometrial thickness of $10 \mathrm{~mm}$ and there was evidence of large heterogeneous lesion in the right adnexa of a size $7.1 \mathrm{~cm} \times 5.1 \mathrm{~cm}$, with sac like structure within which two fetal poles measuring $1.4 \mathrm{~cm}=7$ weeks and five days. Fetal cardiac activity was appreciated. Mild free fluid was seen in the abdomen. An impression of live twin ectopic pregnancy in the right adnexa was made with mild free fluid in an abdomen. A pseudo sac like structure was noticed in endometrial cavity. Both ovaries were visualized separately from the right adnexal mass.

In view of the Twin live ectopic pregnancy she was immediately operated. Intraoperatively, the ampullary part of the right fallopian tube was found showed a sac like structure. A right salphingotomy was done. She was discharged on the $7^{\text {th }}$ day. On cut section of the sac there was evidence of two embryos pathological evaluation of the surgical specimen showed a diamniotic monochorionic twins pregnancy within the fallopian tube and measurement of the twin feti estimated their gestational age at 7 weeks (Figure 1 and Figure 2).

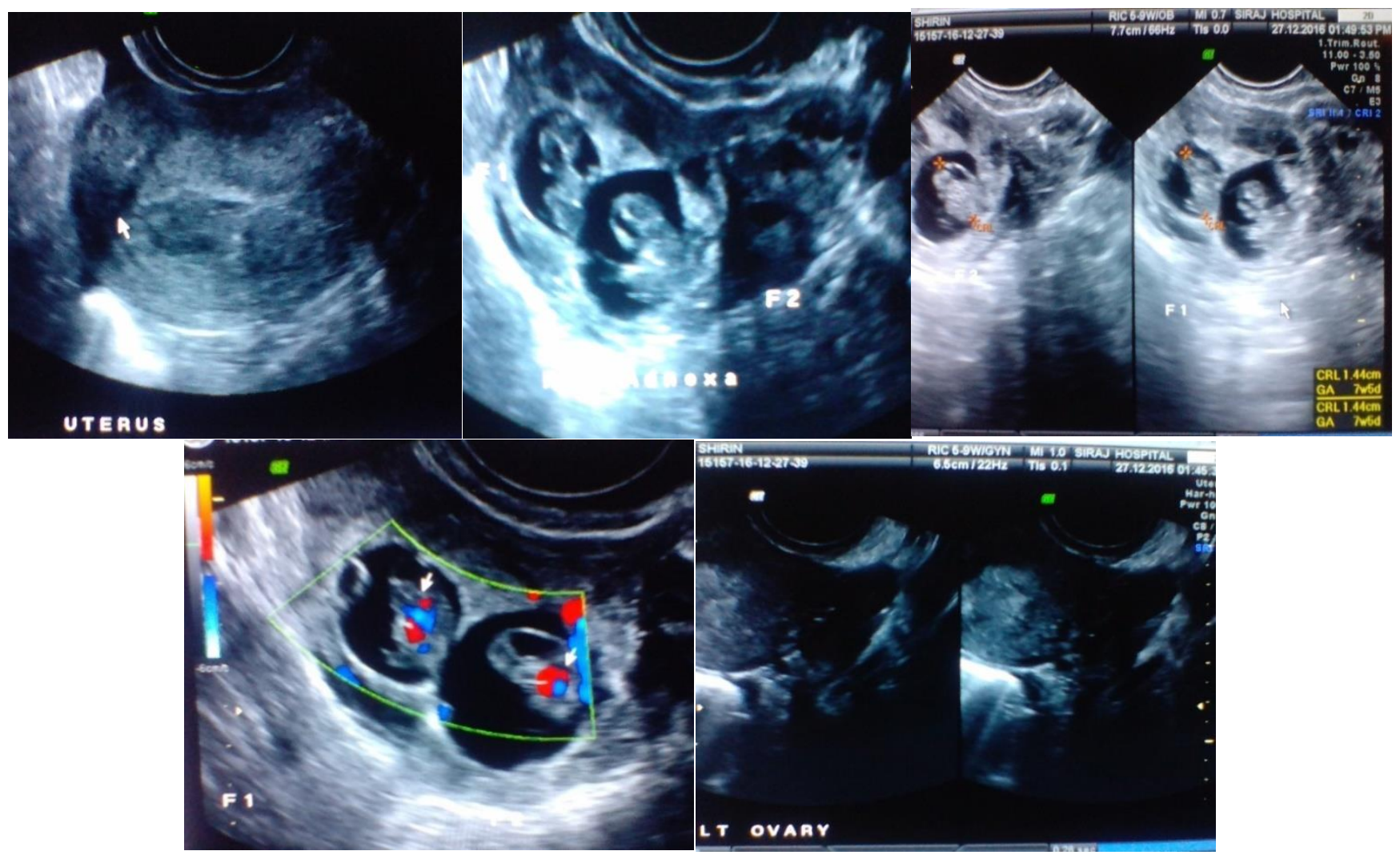

Figure 1: Shows Right adnexal sac with Twin live ectopic gestation of approximately 7 weeks 5 days. No intrauterine pregnancy. Both ovaries are separately visualized. 


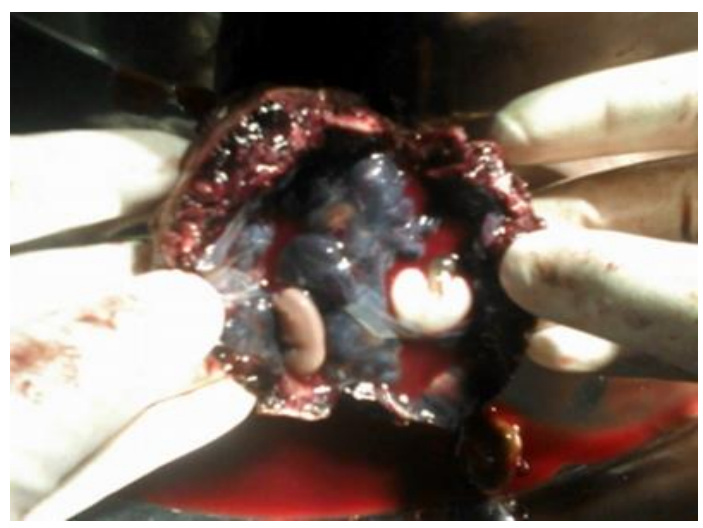

Figure 2: Pathological evaluation of the surgical specimen showed a diamniotic monochorionic twins pregnancy.

\section{Case 2}

A 30-year- old female, G2L1A0 came to casualty with severe pain in abdomen since two days with 6 weeks of amenorrhea and bleeding per vaginum since morning. She had one living issues with full term normal deliveries. Upon examination, her vitals were stable. Abdomen examination was unremarkable. However, her urine pregnancy test was positive.

Spontaneous pregnancy there was no history of induction of ovulation by drugs or artificial reproductive techniques.

\section{The patient was taken for ultra-sonography}

Transvaginal Sonography showed a heterogeneous mass in right adnexa measuring approximately $7.3 \mathrm{~cm} \times 4.6 \mathrm{~cm}$. The mass showed irregular Gestational sac measuring approximately $0.3 \mathrm{~cm}$ corresponding to 5 weeks and another irregular sac measuring $0.5 \mathrm{~cm}$ corresponding to 5 weeks 2 days. Right ovary was visualized separately from this mass.

Left ovary was visualized and appears normal. Moderate hemoperitoneum was seen. Uterus was bulky however there was no intrauterine gestational sac.

The diagnosis of right adnexal ruptured ectopic gestation was made. Preliminary investigations were done and patient was taken up for laparotomy in view of right adnexal mass and hemoperitoneum.

Per operatively gross morphology was: Right adnexal complex mass with moderate hemoperitoneum. Right salpingectomy was performed. Patient's post-operative period was uneventful (Figure 3).

Histopathology report came out to be right adnexal two gestational sacs were noted.

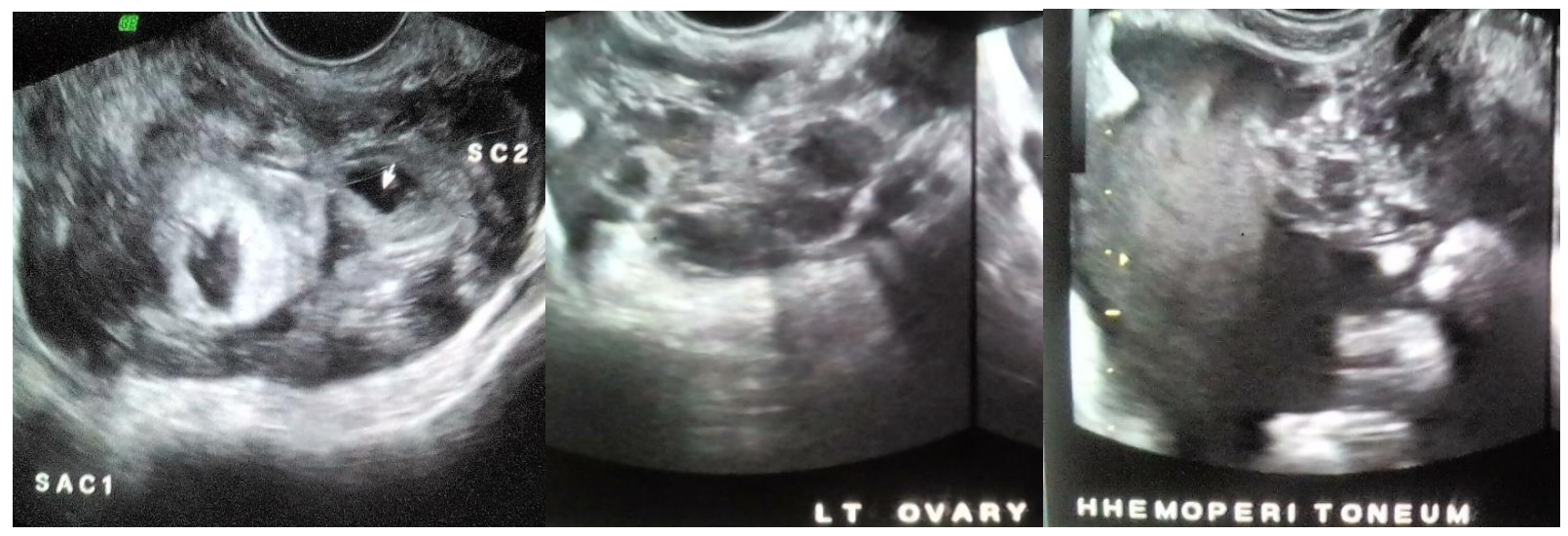

Figure 3: Trans vaginal sonography shows right adnexa heterogenous mass with two irregular sacs and hemoperitoneum.

Left ovary is visualized. 


\section{Case 3}

A 36-year-old female, G3L2A0 came to casualty with severe pain in abdomen since three to four days with 6 weeks of amenorrhea and bleeding per vaginum since morning. Upon examination, her vitals were stable. Abdomen examination was unremarkable. Upon per vaginum examination the cervical movement was tender, the uterus was bulky and soft, and bogginess and tenderness were felt in the both adnexae. However, her urine pregnancy test was positive.

Spontaneous pregnancy there was no history of induction of ovulation by drugs or artificial reproductive techniques.

\section{The patient was taken for ultra-sonography}

Transvaginal Sonography showed a heterogeneous mass in right adnexa with irregular Gestational sac measuring approximately $1.0 \mathrm{~cm}$ corresponding to 5 weeks 5 days Right ovary was visualized separately from this mass.

Left adnexa irregular Gestational sac measuring approximately $0.8 \mathrm{~cm}$ corresponding to 5 weeks 4 days. Left ovary was visualized separately from this mass. Mild to Moderate hemoperitoneum was seen. Uterus was bulky however there was no intrauterine gestational sac.

The diagnosis of Bilateral adnexal ruptured ectopic gestation was made. Preliminary investigations were done and patient was taken up for laparotomy in view of bilateral adnexal masses and hemoperitoneum.

Per operatively gross morphology was: Bilateral adnexal complex masses with moderate hemoperitoneum. Bilateral salpingotomy was performed. Patient's post-operative period was uneventful.

Histopathology report came out to be gestational sacs in bilateral adnexa.

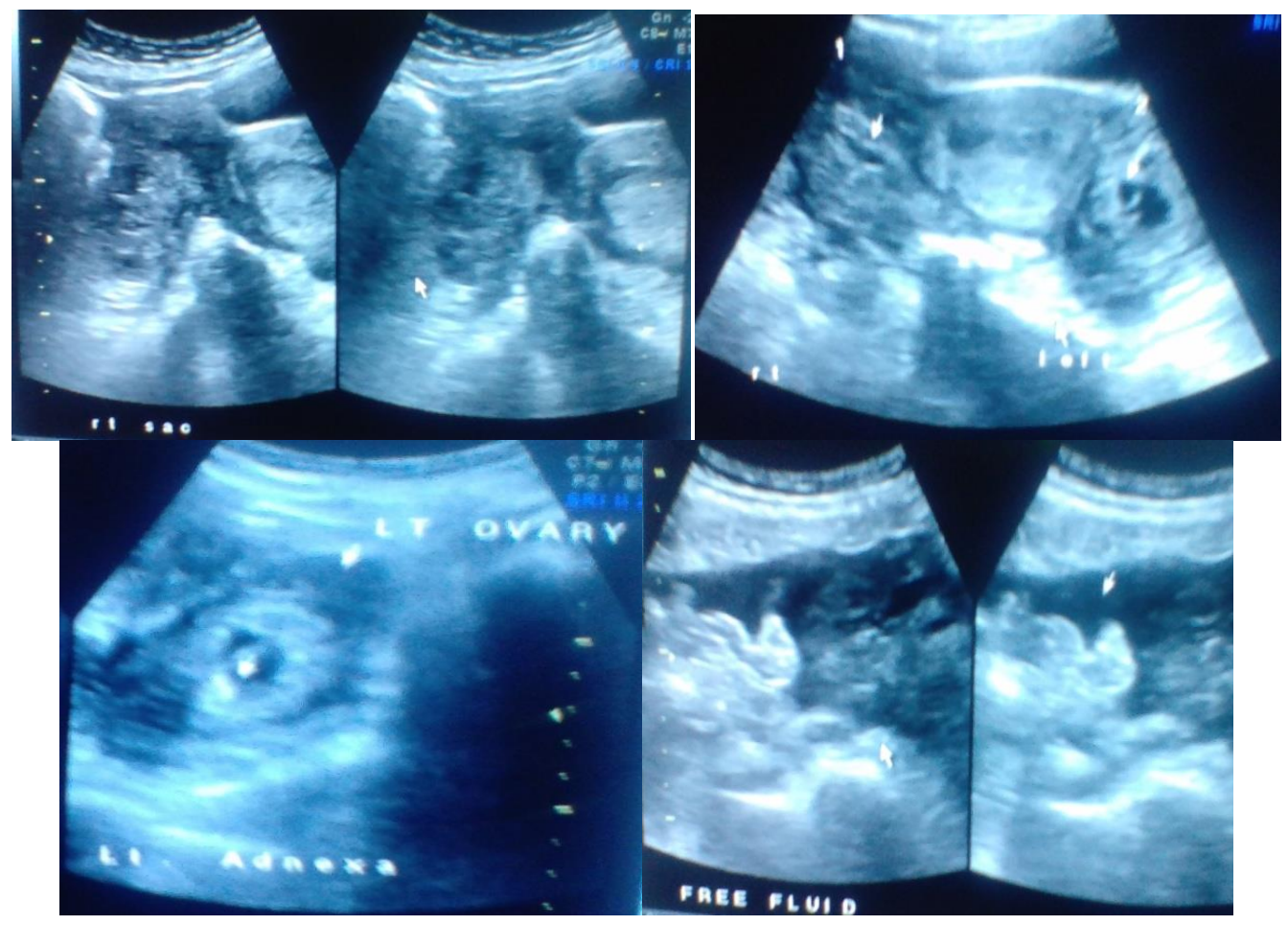

Figure 4: Trans vaginal Sonography shows both adnexae heterogeneous mass with two irregular sacs and hemoperitoneum.

Both ovaries are visualized.

\section{Discussion}

It is found that the incidence of EPs has risen fourfold compared with the rates of 1970 in the United States (from $0.5 \%$ of all 
www.tridhascholars.org | October-2018

pregnancies in 1970 to $2 \%$ in 1992) [4]. However, unilateral tubal twin EPs are still rare and until now about 100 cases have been reported in the literature [5]. Moreover, fetal cardiac activity has been reported in about $<10$ cases [3].

The twin pregnancy is more prevalent among patients with a family history of multiple pregnancies [6]. The incidence of EP is around 1\%-2\% of all pregnancies [7] and the incidence of spontaneous twin pregnancy is 1:90 [8]. However, live unilateral tubal twin EP is a very rare condition and occurs in about 1:125,000 of pregnancies [9]. Several risk factors for tubal EP were identified including active and passive cigarette/tobacco smoking, tubal damage as a result of surgery or infection (particularly Chlamydia trachomatis), and in vitro fertilization [7]. Furthermore, some authors indicated that the number of prior deliveries, EPs, and spontaneous or induced abortions were strongly associated with occurrence of EP [10,11]. No such risk factors were present in our patients. It has been demonstrated that the history of EP leads to an increased recurrence rate of about $10 \%$ and $25 \%$ for one and two/more previous EP, respectively [12].

A history of pelvic pain along with amenorrhea and vaginal bleeding are found in $45 \%$ of EPs [2] and probability of EP in a patient with only abdominal pain and vaginal bleeding is $39 \%$. The likelihood of EP rises to $54 \%$ if the patient has other risk factors, including history of tubal surgery, previous EP, or pelvic inflammatory disease [1].

In addition, ultrasound evaluations have facilitated the early EP diagnosis which may lead to a reduction in maternal mortality and morbidity. Also, use of $\beta$-hCG assay, especially serial measurements, may improve these evaluations. Studies demonstrated that a $\beta$-hCG value of above $1500 \mathrm{mIU} / \mathrm{ml}$ corresponds to an approximately $91.5 \%$ detection of gestational sacs [13]. However, ultrasonographic findings of suspected adnexal mass and free liquid in the Douglas pouch along with an increased a $\beta$-hCG levels, especially in association of risk factors, can help the early diagnosis of EP and reduce the related mortality and morbidity.

Twin-ectopic gestations are extremely rare. More than 100 twin tubal pregnancies have been reported, but 13 cases with cardiac activities demonstrated in both fetuses have been diagnosed [14]. The first case of live twin-ectopic pregnancy was described in 1994 [15]. Unilateral twin ectopic pregnancies occur in 1:200 ectopic pregnancies [16]. Most cases are monochorionic and monozygotic [17]. A rare case of diamniotic dichorionic unilateral twin ectopic pregnancy was reported by Ghike et al. [18] and it appears this patient also had a diamniotic dichorionic twin. They result from the abnormal implantation and maturation of the conceptus outside the endometrial cavity. Diagnosis is made by transvaginal ultrasound and it is crucial to make the diagnosis as soon as possible so that conservative tubal surgery can be planned.

It is reported that the incidence of tubal rupture in was about $32 \%$ and the risk of rupture rises about $2.5 \%$ for every $24 \mathrm{~h}$ period when untreated [19]. Until date, the surgical approach is the most reported option in literature to treat the unilateral tubal twin pregnancies [20]. There have been 4 cases of tubal twin pregnancies ( 3 unilateral, 1 bilateral) that methotrexate treatment has been tried [21-24]. However, Arikan et al. [24] suggested that the nonsurgical treatment may be favored in tubal twin EPs in case of stable maternal vital signs and negative fetal cardiac activities. The tubal twin EP is a major health risk for women of childbearing capacity which may lead to life-threatening complications if not treated properly. Therefore, twin EP must be considered base on physical examination and existence of risk factors and should be carefully looked for on ultrasound scanning, due to the potential mortality and morbidity associated with this condition.

Treatment of an ectopic pregnancy depends on its clinical presentation, size, and complications, and may entail conservative, medical, or surgical intervention. Successful laparoscopic management of tubal twin pregnancy and operative laparoscopic 
www.tridhascholars.org | October-2018

salpingotomy reported [25]. Salphingectomy is the management of choice in cases of large size of ectopic and ruptured ectopic gestation.

In cases of severe hemophilia A with high titer inhibitor, the therapy is not the same as the factor replacement therapy in hemophilia cases without an inhibitor. The inhibitor works on the coagulation factor, deactivating its work so that coagulation becomes difficult. There are two treatments to control bleeding for hemophilia patients with an inhibitor: the first is bypass therapy; the second is inhibitor neutralization therapy, which uses high dose factor administration. The bypass therapy works on the extrinsic and combined coagulation pathway. The bypass therapy uses aPCC, $\mathrm{rF} \phi \mathrm{a}$, and factors $\phi \cdot \imath$ (MC7.10) administration. In the case of children, therapy should be administered with extra caution to prevent thrombophilia and disseminated intravascular coagulation. Tanaka et al. [1] asserts that this therapy is used worldwide according to hemophilia therapy guidelines. Hemophilia patients with a factor inhibitor are categorized into two groups. Patients who receive frequent factor VIII administration and have less than 5 Bethesda Units (BU)/ml of inhibitor are defined as low responders. High responder patients, on the other hand, have over $5 \mathrm{BU} / \mathrm{ml}$ of inhibitor. According to hemophilia therapy guidelines in Japan, first-line treatment for a low responder is inhibitor neutralization therapy, and first-line treatment for a high responder is bypass therapy.

Compartment syndrome was first described by Richard von Volkmann [2]. This syndrome can be caused by trauma, casting, and hemorrhage. The pathophysiology of the disorder is due to a pressure increase within a confined intramuscular compartment space. This can ultimately lead to permanent muscle and nerve damage, limb loss, rhabdomyolysis, and severe functional loss. Hemophilia patients have a high risk of joint hemorrhage, but hemophilia patients who require fasciotomy are relatively rare. If a hemophilia patient with an inhibitor has hemorrhage, he needs a proper diagnosis of this condition, followed by a cooling down position of the affected site, and neutralization therapy or bypass therapy. Fasciotomy should ideally occur within six hours of a compartment syndrome diagnosis. This is supported by Kim et al. [3] who maintain that if a hemophilia patient is diagnosed with compartment syndrome, he needs to undergo fasciotomy within six hours to prevent function loss in the affected area. Moreover, Rorabeck et al. [4] found that if fasciotomy was performed within six hours, there was almost complete recovery of limb function. However, when fasciotomy was performed within 12 hours, normal limb function was regained in only $68 \%$ of patients; when performed after 24 hours, only $8 \%$ of patients regained normal function. If surgical intervention is deemed necessary, it might not only save a limb but also the patient's life. There are few reported cases [5-9] involving patients who needed fasciotomy, but of these cases, the patients had uncontrollable bleeding from the operative site during the postoperative period. In comparing adults to children who underwent fasciotomy, children required more factor administration doses because the factor activation for children is generally shorter than in adults. This is why there is more difficulty in controlling post-operative bleeding in children. In this particular case, the patient received rFVIIa as well as aPCC in bypass therapy before fasciotomy. However, the postoperative bleeding was still difficult to control even though the patient received bypass therapies as well as transfusions. The Authors who have reported on hemophilia cases with fasciotomy remark that it takes time to control the bleeding post-operation, as was true in this case. Hemophilia patients who receive fasciotomy, particularly in the case of children, require special care during hospitalization so that postoperative bleeding can be managed and controlled.

\section{Conclusion}

A spontaneous twin tubal pregnancy can occur in patients who have no known predisposing factor. Early diagnosis has made 
www.tridhascholars.org | October-2018

this disorder amenable to appropriate treatment. The high-resolution transvaginal sonography is very helpful in the diagnosis of this condition. Twin-ectopic gestations are extremely rare but there are treatment options. These have typically been classified as either conservative or surgical.

Authors' Note: The patient's consent was obtained for publication of this case report.

Conflict of Interest: The author(s) declared no potential conflicts of interest with respect to the research, authorship, and/or publication of this article.

Funding: The author(s) received no financial support for the research, authorship, and/or publication of this article.

\section{References}

1. Lozeau AM and Potter B (2005) Diagnosis and management of ectopic pregnancy. American Family Physician Journal 72(9): 1707-1714.

2. Dede M, Gezginç K, Yenen M, et al. (2008) Unilateral tubal ectopic twin pregnancy. Taiwanese Journal of Obstetrics and Gynecology 47(2): 226-228.

3. Eddib A, Olawaiye A, Withiam-Leitch M, et al. (2006) Live twin tubal ectopic pregnancy. International Journal of Gynecology \& Obstetrics 93(2): 154-155.

4. Hois EL, Hibbeln JF, Sclamberg JS (2006) Spontaneous twin tubal ectopic gestation. Journal of Clinical Ultrasound 34(7): 352-355.

5. Della-Giustina D and Denny M (2003) Ectopic pregnancy. Emergency Medicine Clinics of North America 21(3): 565-584.

6. Kullima AA, Audu BM, Geidam AD (2011) Outcome of twin deliveries at the University of Maiduguri Teaching Hospital: A 5-year review. Nigerian Journal of Clinical Practice 14(3): 345-348.

7. Shaw JLV, Dey SK, Critchley HOD et al. (2010) Current knowledge of the etiology of human tubal ectopic pregnancy. Human Reproduction Update 16(4): 432-444.

8. Kazandi M and Turan V (2011) Multiple pregnancies and their complications. Journal of Turkish Society of Obstetrics and Gynecology 8(1): 21-24.

9. Parker J, Hewson AD, Calder-Mason T et al. (1999) Transvaginal ultrasound diagnosis of a live twin tubal ectopic pregnancy. Australasian Radiology 43(1): 95-97.

10. Bouyer J, Coste J, Shojaei T, et al. (2003) Risk factors for ectopic pregnancy: a comprehensive analysis based on a large case-control, population-based study in France. American Journal of Epidemiology 157(3): 185-194.

11. Barnhart KT (2009) Ectopic pregnancy. New England Journal of Medicine 361(4): 379-387.

12. Seeber BE and Barnhart KT (2006) Suspected ectopic pregnancy. Obstetrics \& Gynecology 107(2): 399-413.

13. Barnhart K, Mennuti MT, Benjamin I, et al. (1994) Prompt diagnosis of ectopic pregnancy in an emergency department setting. Obstetrics and Gynecology 84(6): 1010-1015.

14. Summa B, Meinhold-Heerlein I, Bauerschlag DO, et al. (2009) Early detection of a twin tubal pregnancy by Doppler sonography allows fertility-conserving laparoscopic surgery. Archives of Gynecology and Obstetrics 279(1): 87.

15. Gualandi M and Steemers N (1994) First reported case of preoperative ultrasonic diagnosis and laparoscopic treatment of unilateral, twin tubal pregnancy. Revue Francaise de Gynecologie et d'Obstetrique 89(3): 134-136. 
www.tridhascholars.org | October-2018

16. Breen JL (1970) A 21 year survey of 654 ectopic pregnancies. American Journal of Obstetrics \& Gynecology 106(7): 1004-1019.

17. Storch MP and Petrie RH (1976) Unilateral tubal twin gestation. American Journal of Obstetrics \& Gynecology 125(8): 1148.

18. Ghike S, Somalwar S, Mitra K, et al. (2011) Unilateral twin ectopic pregnancy (diamniotic-dichorionic): a rare case. Journal of SAFOG with DVD 3(2): 103-105.

19. Bickell NA, Bodian C, Anderson RM et al. (2004) Time and the risk of ruptured tubal pregnancy. Obstetrics \& Gynecology 104(4): 789-794.

20. Tam T and Khazaei A (2009) Spontaneous unilateral dizygotic twin tubal pregnancy. Journal of Clinical Ultrasound 37(2): 104-106.

21. Fernandez H, Bourget P, Lelaidier C, et al. (1993) Methotrexate treatment of unilateral twin ectopic pregnancy: case report and pharmacokinetic considerations. Ultrasound in Obstetrics and Gynecology 3(5): 357-359.

22. Marcovici I and Scoccia B (1997) Spontaneous bilateral tubal ectopic pregnancy and failed methotrexate therapy: a case report. American Journal of Obstetrics \& Gynecology 177(6): 1545-1546.

23. Karadeniz RS, Dilbaz S and Özkan SD (2008) Unilateral twin tubal pregnancy successfully treated with methotrexate. International Journal of Gynecology \& Obstetrics 102(2): 171-171.

24. Arikan DC, Kiran G, Coskun A et al. (2011) Unilateral tubal twin ectopic pregnancy treated with single-dose methotrexate. Archives of Gynecology and Obstetrics 283(2): 397-399.

25. Gassibe EF and Gassibe E (1996) Laparoscopic management of ruptured tubal twin pregnancy. The Journal of the American Association of Gynecologic Laparoscopists 3(4 Supplement): S15-S15. 